\title{
Metallic ferroelectricity induced by anisotropic unscreened Coulomb interaction in $\mathrm{LiOsO}_{3}$
}

\author{
H. M. Liu ${ }^{1}$, Y. P. Du ${ }^{1}$, Y. L. Xie ${ }^{1}$, J. -M. Liu ${ }^{1}$, Chun-Gang Duan ${ }^{2}$ and Xiangang Wan用 \\ ${ }^{1}$ National Laboratory of Solid State Microstructures and Department of Physics, Nanjing University, Nanjing 210093, China \\ ${ }^{2}$ Key Laboratory of Polar Materials and Devices, Ministry of Education, \\ East China Normal University, Shanghai 200062, China
}

(Dated: August 8, 2018)

\begin{abstract}
As the first experimentally confirmed ferroelectric metal, $\mathrm{LiOsO}_{3}$ has received extensive research attention recently. Using density-functional calculations, we perform a systematic study on the origin of the metallic ferroelectricity in $\mathrm{LiOsO}_{3}$. We confirm that the ferroelectric transition in this compound is order-disorder like. In addition, we find that the distribution of the conduction electron is extremely anisotropic, which suggests that the electric screening ability is also highly anisotropic. Then, by doing electron screening analysis, we unambiguously demonstrate that the long range ferroelectric order in $\mathrm{LiOsO}_{3}$ results from the incomplete screening of the dipole-dipole interaction along the nearest neighboring Li-Li chain direction. We therefore conclude highly anisotropic screening and local dipole-dipole interactions are two most important keys to form $\mathrm{LiOsO}_{3}$-type metallic ferroelectricity.

PACS numbers: 71.20.-b, 72.80.Ga, 77.80.B-, 61.50.Ah
\end{abstract}

The ferroelectric (FE) instability can be explained by a delicate balance between short-range elastic restoring forces supporting the undistorted paraelectric $(\mathrm{PE})$ structure and long-range Coulomb interactions favoring the FE phase [1]. Itinerant electrons can screen the electric fields and inhibit the electrostatic forces, metallic systems are thus not expected to exhibit ferroelectric like structural distortion. Despite the incompatibility, using a phenomenological theory, Anderson and Blount proposed in 1965 that metals can break inversion symmetry 2]. They found that the FE metal is possible through a continuous structural transition accompanied by the appearance of a polar axis, and the disappearance of an inversion center [2]. In $2004, \mathrm{Cd}_{2} \mathrm{Re}_{2} \mathrm{O}_{7}$ had been proposed as a rare example of ferroelectric metals [3], however, it was found that although this compound exhibits a second order phase transition to a structure that lacks inversion symmetry, a unique polar axis could not be identified [4], which does not fit the criteria about the FE metal.

Very recently, the first convincing success was achieved experimentally in $\mathrm{LiOsO}_{3}$ [1]. $\mathrm{LiOsO}_{3}$ remains metallic behavior while it undergoes a second-order phase transition from the high temperature centrosymmetric $R \overline{3} c$ to a FE-like $R 3 c$ structure at $T_{s}=140 \mathrm{~K}[1]$. Neutron and $\mathrm{x}$ ray diffraction studies showed that the structural phase transition involves the displacements of $\mathrm{Li}$ ions accompanying also a slight shift of $\mathrm{O}$ ions [1]. The electronic structure and lattice instability were studied by several groups 6 - 8$]$. It was found that the local polar distortion in $\mathrm{LiOsO}_{3}$ is solely due to the instability of the $A$-site $\mathrm{Li}$ ion [6 8]. The importance of the Coulomb interaction among $5 d$ electrons and the hybridization between oxygen $p$ orbitals and Os empty $e_{g}$ orbitals has also been emphasized by Giovannetti and Capone [8]. Despite these efforts devoted to understanding the origin of the FE like structural transition in this metallic system, there are still two fundamental issues have not been clearly clarified. The first is the origin of the ferroelectric instability: is it displacive or order-disorder? Second, as FE-like phase transition of $\mathrm{LiOsO}_{3}$ occurs at a relatively high temperature $(140 \mathrm{~K})$, how can these local dipoles lined up to form long-range order, as if there is no conduction electrons to screen the dipole interactions.

In this paper, based on the density functional theory (DFT) calculations, we reveal the microscopic mechanism for the FE-like structural transition in $\mathrm{LiOsO}_{3}$. Our study shows that different from other $5 d$ transition metal oxides 9 15], the spin-orbital coupling (SOC) and the electronic correlation do not play important role in $\mathrm{LiOsO}_{3}$. Our comprehensive potential surface calculations suggest that the structural transition is orderdisorder like. The most striking finding is that the electric screening in $\mathrm{LiOsO}_{3}$ is highly anisotropic despite its metallic nature. Consequently, the dipole-dipole interactions are unscreened along certain directions, which results in the long range $\mathrm{FE}$ order at considerably high temperature. This is in sharp contrast to the case in the displacive type FE compounds, where the FE structural transition is usually driven by hybridization or lonepair [16] and consequently the change of electric dipole (namely the atomic motion) will modify the valence band significantly. If such displacive type FE compounds become metallic, the interactions between their electric dipoles will be strongly screened out, and the metallic FE phase is highly unlikely to occur.

Before the formal presentation of the calculated results, we would like to first discuss our strategy to study the electric screening effect. As is well known, the major difference between the insulator and metal is that there are free non-localized electrons in metals, whereas in in- 
sulators there are only bound electrons. Consequently, electrostatic forces will be strongly screened by the itinerant electron in the metallic system. The screening effect actually can be described as the electron charge difference induced by a perturbation such as the change of the dipole or external electric field [17]. Yet seldom efforts have been carried out to study the screening effect in the bulk metal, as people generally believe there is no macroscopic electric field inside metals. But in current study we will control the formation of local dipoles in the bulk metal and study the charge difference (response) caused by the change of dipole. This provides a explicit picture on the exact behavior of the screening effect in metallic systems.
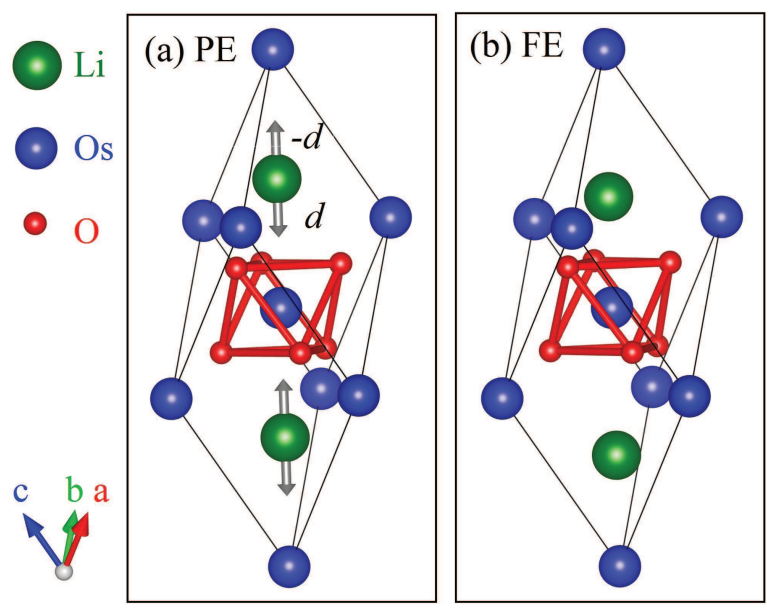

FIG. 1: (Color online) Primitive unit cell of (a) PE and (b) FE phases of $\mathrm{LiOsO}_{3}$. The green, blue and red balls are the $\mathrm{Li}$, Os and $\mathrm{O}$ ions respectively. $d$ and $-d$ corresponds to the displacements of $\mathrm{Li}$ ions along the polar axis.

Our first-principles calculation is performed using the VASP (Vienna ab-initio simulation package) code [18, 19]. The results presented in the following are obtained by using the GGA (generalized gradient approximation) PBE (Perdew-Becke-Erzenhof) function [20], a $20 \times 20 \times 20$ mesh for the Brillouin-zone sampling and 500 $\mathrm{eV}$ for cut-off of the plane-wave basis set. The effect of exchange-correlation function, pseudopotential, cut-off value has been carefully checked, and some of the results are shown in the Supplementary Materials [21].

There are 10 atoms in the primitive unit cell of $\mathrm{LiOsO}_{3}$. The atomic arrangements are sketched in Fig. 1. In the $R \overline{3} c$ PE structure, the Os atoms are at the centers of the oxygen octahedrons, while Li atoms are centered between two adjacent Os atoms along the polar axis on average. Using the experimental lattice parameters, we optimize all independent internal atomic coordinates of the FE structure until the Hellman-Feynman forces on every atom are converged to less than $1 \mathrm{meV} / \AA$, the optimized internal atomic coordinates are listed in Tab. [1. the experimental PE and FE structure had also been pre-

\begin{tabular}{ccc}
\hline \hline & Atom & Position \\
\hline \multirow{2}{*}{ PE } & Li & $(0.25,0.25,0.25)$ \\
& Os & $(0,0,0)$ \\
& O & $(0.8798,-0.3798,0.25)$ \\
\hline \multirow{2}{*}{ FE(Expt. $)$} & Li & $(0.2147,0.2147,0.2147)$ \\
& Os & $(0,0,0)$ \\
& O & $(0.8785,-0.3837,0.2627)$ \\
FE(Calc. $)$ & Li & $(0.2149,0.2149,0.2149)$ \\
& Os & $(0,0,0)$ \\
\hline \hline
\end{tabular}

TABLE I: Atomic positions (in primitive rhombohedral coordinates) in $\mathrm{PE}$ and FE phases. The experimental results are from Ref. [1].

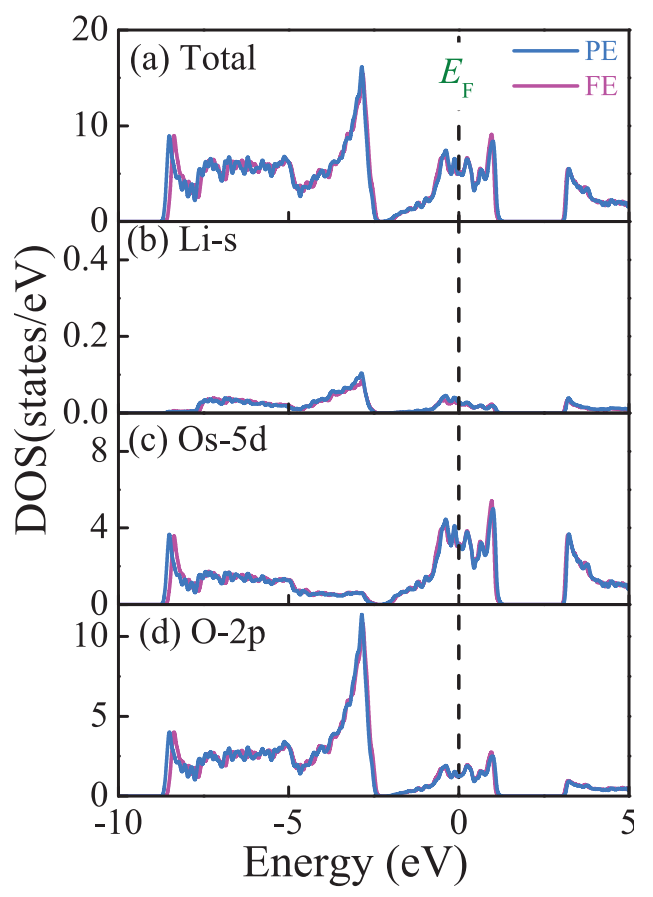

FIG. 2: (Color online) (a) The total DOS patterns of $\mathrm{LiOsO}_{3}$ in $\mathrm{PE}$ (blue) and FE(pink) phases. The partial DOS of (b) Li$1 s$ (c) Os-5d and (d) O-2p states in PE(blue) and FE(pink) phases respectively. The Fermi energy is positioned as zero.

sented at Tab. I for comparison. The calculated results coincide with previous experimental and calculated results [1, 6 8], the FE structural phase transition mainly involves the displacements of $\mathrm{Li}$ atoms: Li atoms shift along the polar axis about $d \sim 0.47 \AA$ from the mean positions of the PE phase (see grey arrow $d$ in Fig. 11(a)) and $\mathrm{O}$ atoms slightly displace about $0.056 \AA$ [1, 6, 8].

Based on calculated lattice structure, we first perform standard GGA calculation to see the basic features of the electronic structure of $\mathrm{LiOsO}_{3}$. We show the total and partial density of states (DOS) in Fig. 2. Our results are consistent with previous work [1, 6]. The energy range, -9.0 to $-2.4 \mathrm{eV}$ is dominated by $\mathrm{O}-2 p$ orbital with an ad- 
ditional contribution from the Os-5 5 state indicating hybridization between them. Li is highly ionic and its bands are far from the Fermi level. The Os atom is octahedrally coordinated by six $\mathrm{O}$ atoms, making the Os $5 d$ band split into the $t_{2 g}$ and $e_{g}$ states, and the $t_{2 g}$ bands are located from -2.2 to $1.2 \mathrm{eV}$, as shown in Fig. 2(c). Due to the extended nature of $5 d$ states, the crystal splitting between $t_{2 g}$ and $e_{g}$ states is large, and the $e_{g}$ states are located about $3.0 \mathrm{eV}$ higher than the Fermi energy and disperse widely. As shown in the comparison of DOS of PE and $\mathrm{FE}$, the electronic structures almost do not change during the phase transition, which is consistent with the previous theoretical work [6]. It is worthy to mention that this is quite different from prototype FE systems such as $\mathrm{BaTiO}_{3}$, in which hybridization is necessary for the FE phase transitions 22 24]. Thus we think the hybridization is not the driving force for the structural instability in $\mathrm{LiOsO}_{3}$.

It is well known that the SOC of $5 d$ electrons is very strong [25] and usually changes the $5 d$ band dispersion significantly, as demonstrated in $\mathrm{Sr}_{2} \mathrm{IrO}_{4}$ [9], pyrochlore iridates and spinel osmium [10, 11]. In the case of $\mathrm{LiOsO}_{3}$, as shown in Fig. 2, the O- $2 p$ orbitals are almost fully occupied, while the bands of $\mathrm{Li}$ are mainly empty, thus Os occurs in its $5+$ valence state and there are basically three electrons in its $t_{2 g}$ band. Since $t_{2 g}$ band is half filled, it is natural to expect the effect of SOC to be small despite the large strength of SOC 12 . This has been confirmed by the comparison of the band structures obtained in the presence and absence of SOC (see Fig.S 1 in Supplemental Material [21]).

Although the $5 d$ orbitals are spatially extended, it has been found that the electronic correlations are important for $5 d$ transition metal oxides [9-13]. Here, we estimate the Sommerfeld coefficient based on the numerical DOS at Fermi level. Our numerical result (6.1 $\mathrm{mJ} \mathrm{mol}^{-1} \mathrm{~K}^{-2}$ ) is just slightly less than that of experimental one $\left(\gamma=7.7 \mathrm{~mJ} \mathrm{~mol}^{-1} \mathrm{~K}^{-2}\right)$ [1] which indicates the electronic correlation is not important for $\mathrm{LiOsO}_{3}$. This is in sharp contrast with the iridates, spinel osmium [9 11], and $\mathrm{NaOsO}_{3}$ 12].

One fundamental issue about this system is the mechanism for the ferroelectric instability, is it displacive or order-disorder? Using comprehensive total energy calculations, we now try to solve this issue. Following the common procedure used in the study of FE structures, we first calculate the potential energy profile along different displacive soft modes, i.e the evolution paths from the PE to FE structures. The results, as shown in Fig. 3, suggest that the energy difference between the PE and FE structures is majorally contributed by $\mathrm{Li}$ ions movements. The depth of double wells resulting from the motion of $\mathrm{Li}$ ions only and both of the $\mathrm{Li}$ and $\mathrm{O}$ ions are 27 and $44 \mathrm{meV}$, respectively, which is in consistent with several previous works $[6,[8]$. Note that we find it is important to adopt the optimized structure to obtain the

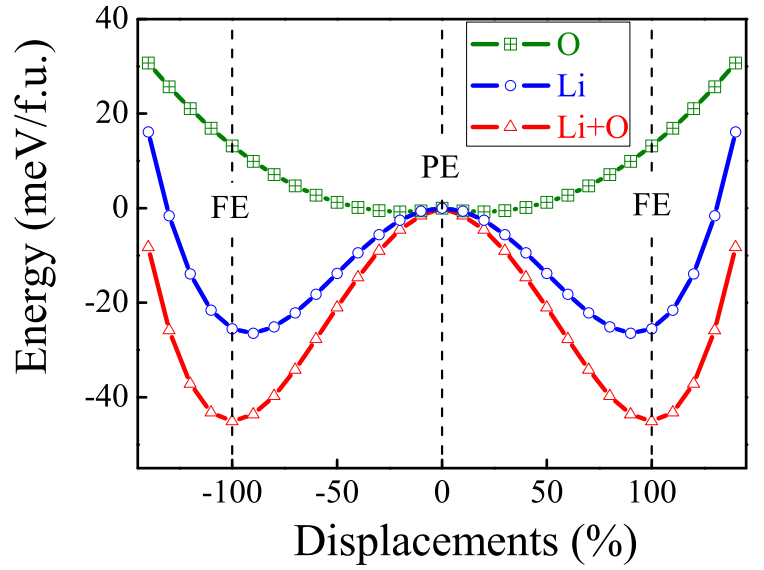

FIG. 3: (Color online) The olive, blue and red curves represents the potential energy changes with respect to $\mathrm{O}$ displacements only, Li displacements only and the coupled displacements of the $\mathrm{Li}$ and $\mathrm{O}$ ions. The total energy and displacements of PE states are set as zero. The displacements of corresponded FE states are set as $100 \%$.

correct potential energy surfaces. Adopting experimental coordinates will obtain unreasonable results, as the well depth caused by the sole Li ion movements is even larger than that of considering both the $\mathrm{Li}$ and $\mathrm{O}$ moments (see Fig.S2 in Supplemental Material [21]).

Nevertheless, we notice that the experimental transition temperatures of $\mathrm{LiOsO}_{3}$ is $140 \mathrm{~K}$ and much lower than the depths of double wells. This indicates the transition in $\mathrm{LiOsO}_{3}$ is most probably order-disorder like [26]. This may also explain the experimentally observed incoherent charge transport above the transition temperature 1], which is possibly caused by the scattering induced by disorder of Li off-center displacement. For order-disorder transition, the $\mathrm{Li}$ atoms oscillate between the double wells and the potential wells remain basically unchanged throughout the phase transition, thus we expect there is no softening mode in the Raman spectra of $\mathrm{LiOsO}_{3}$.

As mentioned above, the $\mathrm{Li}$ ions in $\mathrm{LiOsO}_{3}$ favor an off-center displacement and form local dipoles as shown in Fig. 1. Thus it is a puzzle why the local electric dipoles in different unit cell can interact with each other and form a long range order at $140 \mathrm{~K}$, noticing that the distance between them is far (even the nearest neighbor dipole distance is larger than $3.5 \AA$ ) and the DOS at the Fermi level is rather large (Fig. 22). We find that the bands located below $-10 \mathrm{eV}$ are quite narrow and have negligible hybridization with other bands. The electrons at these bands are tightly bounded with the ion, thus almost do not change with the motion of Li ion, namely these electrons almost have no contribution to the electric screening effect. On the other hand, the displacements of $\mathrm{Li}$ ions just slightly affect the Os- $5 d$ and O- $2 p$ electrons as shown in Fig. 2. To have a straightforward view of the charge distribution, we sketched the electron densi- 
(a)

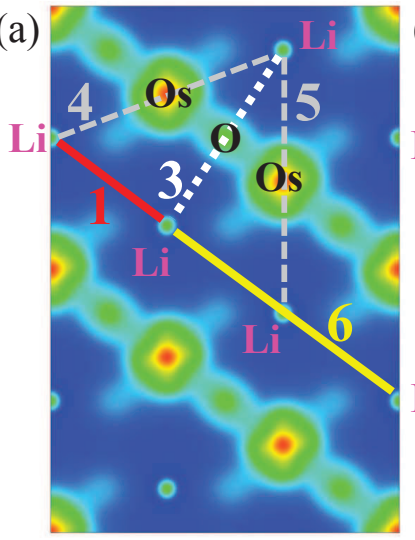

(b)

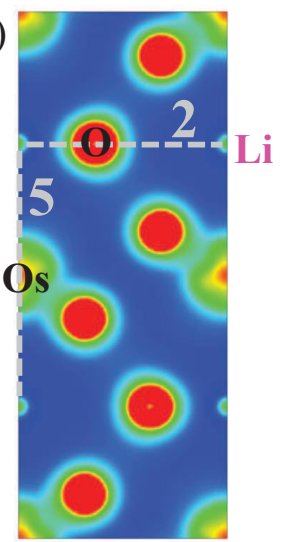

(c) 1

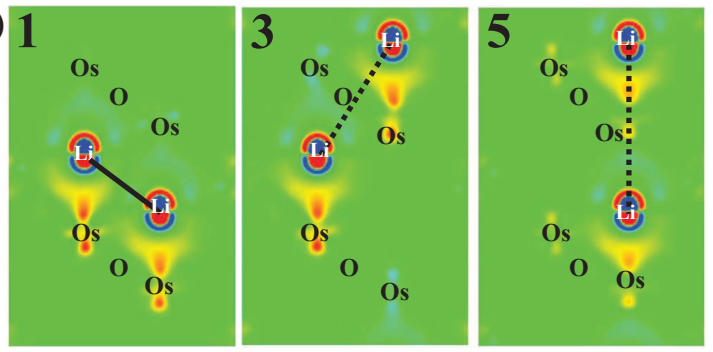

FIG. 4: (Color online) Partial electron densities contour maps for $\mathrm{LiOsO}_{3}$ taken through (a) $\left[\begin{array}{lll}1 & -1 & 0\end{array}\right]$ and (b) [ $\left.\begin{array}{lll}2 & -1 & 0\end{array}\right]$ plane. Contour levels shown are between 0 (blue) and $0.3 \mathrm{e} / \AA^{3}$ (red). (c) Charge density difference between FE and PE structures for Li pair 1, 3 and 5 through [ $\left.\begin{array}{lll}1 & -1 & 0\end{array}\right]$ plane. See text for details. Contour levels shown are between -0.004 (blue) and $0.004 \mathrm{e} / \AA^{3}$ (red).

ties arising from states between $-10 \mathrm{eV}$ and the Fermi level in Fig. 4(a,b). There are two distinctive characters in these two figures. One is that the electronic density is relevant high between the Os and $\mathrm{O}$ ions, which again indicates the strong hybridization between Os- $5 p$ and $\mathrm{O}-2 p$ states. The second is that there is almost no conduction charge at all in a relative large space around the $\mathrm{Li}$ ions, i.e. the Li ion is literally bare ion. We will demonstrate later that the later character directly results in incomplete electric screening of dipole-dipole interactions and forms long range dipole ordering.

Following the previously described procedure, we then demonstrate the screening effect by doing charge difference calculation. Since the FE like transition basically involves displacements of $\mathrm{Li}$ ion, the change of local dipole can be approximated by the Li movement from the PE structure and the dipole interactions can be labeled as Li-Li pairs. Fig. 固, we use symbol 1, 2, 3 etc. to denote the $\mathrm{Li}-\mathrm{Li}$ pairs with the first, second and third nearest distance between them. As is clear in Fig. 4(a), there is almost no conduction charge distribution between pair 1 (red solid line), thus it is natural to expect the screening effect for the nearest dipole-dipole interaction is small. This had been confirmed by the calculation of the charge difference induced by the Li motions. We first construct

\begin{tabular}{|c|c|c|c|c|c|c|}
\hline $\mathrm{NN}$ & $1 \mathrm{st}$ & $2 \mathrm{nd}$ & $3 \mathrm{rd}$ & 4th & 5 th & 6 th \\
\hline$d_{i}(\ddot{\mathrm{A}})$ & 3.66 & 5.06 & 5.28 & 6.25 & 6.61 & 7.32 \\
\hline$J_{i}(\mathrm{meV})$ & -4.2 & -0.16 & -1.9 & -0.17 & -0.06 & -0.27 \\
\hline
\end{tabular}

TABLE II: The distances $d_{i}(i=1-6)$ and coupling parameters $J_{i}(i=1-6)$ between $i$ th $\mathrm{Li}$ ion pairs.

a $3 \times 3 \times 1$ supercell containing 270 atoms in hexagonal phase. The PE structure is taken as background. Then we move $\mathrm{Li}$ ions in pair 1 to their $\mathrm{FE}$ positions, and all other atoms are fixed. In this way we can see what happened when the two local dipoles are formed. The charge density change (namely $\Delta \rho=\rho_{F E}-\rho_{P E}$ ) is then plotted in the left panel of Fig. 4(c), which clearly demonstrates the screening of these two dipoles. As can be seen from this plot, significant conduction electron responses only occur around Os ions, and they form local dipoles to against the Li dipoles, this is exactly the screening effect which is expected in metallic systems. Whereas the charge distribution near $\mathrm{O}$ remains almost unchanged, indicating the Os-5 $d$ and O-2p hybridization is neither important for the electric dipole interaction, nor will be affected by the Li dipoles. The most interesting thing is, as shown in the left panel of Fig. 4(c), there is almost no modification of charge distribution between pair 1 at all. This clearly demonstrates that dipole interaction between pair 1 is only slightly screened. We then apply the same strategy to study other Li pairs. For pair 2 (Fig. 4(b)), as there is $\mathrm{O}$ atom between two $\mathrm{Li}$ ions, we observe noticeable screening to prevent the direct dipole interaction. Yet for pair 3, the dipole interaction is again not fully screened as shown in the middle panel of Fig. 4(c). Detailed analysis indicates that this is because this Li pair is $0.65 \AA$ away from the $\mathrm{O}$ ion plane. For other pairs, as there is either $\mathrm{O}$ or Os atoms between two Li ions, the electric screening effect is strong. The example of pair 5 , which is also at the $\left[\begin{array}{lll}1 & -1 & 0\end{array}\right]$ plane like pair 1 and 3, is shown in the right panel of Fig. 4(c) for comparison.

Above we have provided a qualitative picture about why locale dipole interactions are not fully screened in $\mathrm{LiOsO}_{3}$. To give a more quantitative explanation, we try to estimate the interaction strength between the local electric dipole moments. Again using the above adopted supercell, we obtain coupling constant $J_{i}(i=1-6)$ between the $i$ th Li pairs from the energy difference between the local $\mathrm{FE}$ and antiferroelectric (AFE) states $(i$ th $\mathrm{Li}$ pairs are AFE ordered), i.e $J_{i}=\left[\mathrm{E}_{F E}-\mathrm{E}_{A F E}\right] / 2$. The LiLi distances $d_{i}$ of each pair and the obtained interaction parameter $J_{i}$ are listed in Table II. Consistent with the above screening discussions, $J_{1}$ and $J_{3}$ are much larger than all of other interactions, indicating the dipole interactions are highly anisotropic. Despite $d_{6}$ is longer than $d_{2}, d_{4}$ and $d_{5}, J_{6}$ is considerably larger than $J_{2}, J_{4}$ and $J_{5}$ 
as shown in Table II, which also indicate the anisotropic screening effect in this metallic compound.

To show these dipole interaction parameters obtained in above procedure are reasonable, we perform the Monte-Carlo (MC) simulations using an effective Isinglike Hamiltonian: $H=\sum_{i} J_{i} D_{m} D_{n}, J_{i}$ is the coupling constant between dipole moments $D_{m}$ and $D_{n}$. The obtained phase transition temperature is $210 \mathrm{~K}$ with only $J_{1}$ considered and $330 \mathrm{~K}$ with both $J_{1}$ and $J_{3}$ considered, which is reasonably higher than the experimental $T_{s}(140 \mathrm{~K})$, and the overestimation may come from the rigid dipole model used in our MC simulations. This, again, show that our explanation on the mechanism of the lineup of local dipoles in metallic $\mathrm{LiOsO}_{3}$ is self consistent.

In summary, we use a straightforward way to study the electric screening effect in bulk metallic systems. We conclude that microscopic mechanism of metallic ferroelectricity in $\mathrm{LiOsO}_{3}$ is not related spin-orbital interaction or electronic correlation, as one may expect for $5 d$ system. It is the highly inhomogeneous charge distribution in this metallic system plays crucial roles in the ferroelectric-like structural transition. The highly ionized Li atoms are easy to form local dipoles as a consequence of the Coulomb interactions. The screening in this compound, different from our previous thought for a metallic system, is strongly anisotropic. Therefore local dipole interactions along certain directions are not fully screened and result in the dipole ordering below rather high temperature. We also want to emphasis that the above picture implied that the structural phase transition should be order-disorder instead of displacive type, which is also supported by our potential energy surface calculations. This is because the displacive-type ferroelectric transition occurring in $\mathrm{ABO}_{3}$ perovskite structures are general triggered by the hybridization between the $\mathrm{B}$ and $\mathrm{O}$ electronic states or the lone-pair in A site. In either case the change of the electric dipole will modify the valence band considerably. Therefore in displacive system the dipole-dipole interaction will be strongly screened out in the metallic phase.

HML is thankful to Z. Z. Du and Y. L. Wang for helpful discussions. The work was supported by the National Key Project for Basic Research of China (Grants No. 2011CB922101, 2014CB921104), NSFC under Grants No. 91122035, 11174124, 51431006, 11374147, 51332006 and 61125403 . The project is also funded by Priority Academic Program Development of Jiangsu Higher Education Institutions.

\footnotetext{
* Corresponding author: xgwan@nju.edu.cn

[1] W. Cochran, Adv. Phys. 9, 387 (1960).

[2] P. W. Anderson, and E. I. Blount, Phys. Rev. Lett. 14,
}

$217(1965)$

[3] I. A. Sergienko, V. Keppens, M. McGuire, R. Jin, J. He, S. H. Curnoe, B. C. Sales, P. Blaha, D. J. Singh, K. Schwarz, and D. Mandrus, Phys. Rev. Lett. 92, 065501 (2004).

[4] V. Keppens, Nature Mater. 12, 952 (2013).

[5] Y. Shi, Y. Guo, X. Wang, A. J. Princep, D. Khalyavin, P. Manuel, Y. Michiue, A. Sato, K. Tsuda, S. Yu, M. Arai, Y. Shirako, M. Akaogi, N. Wang, K. Yamaura and A. T. Boothroyd, Nature Mat. 12, 1024 (2013).

[6] H. Sim, and B. G. Kim, Phys. Rev. B 89, 201107(R) (2014).

[7] H. J. Xiang, arxiv 1312.4225 (2013).

[8] G. Giovannetti and M. Capone, arxiv 1404.7705 (2014).

[9] B. J. Kim, H. Jin, S. J. Moon, J.-Y. Kim, B.-G. Park, C. S. Leem, J. Yu, T. W. Noh, C. Kim, S.-J. Oh, J.-H. Park, V. Durairaj, G. Cao, and E. Rotenberg, Phys. Rev. Lett. 101, 076402 (2008); H. Watanabe, T. Shirakawa, and S. Yunoki, Phys. Rev. Lett. 105, 216410 (2010).

[10] X. Wan, A. M. Turner, A. Vishwanath, and S. Y. Savrasov, Phys. Rev. B 83, 205101 (2011); B. J. Yang and Y. B. Kim, Phys. Rev. B 82, 085111 (2010).

[11] X. Wan, A. Vishwanath, and S. Y. Savrasov, Phys. Rev. Lett. 108, 146601 (2012).

[12] Y. Du, X. Wan, L. Sheng, J. Dong, and S. Y. Savrasov, Phys. Rev. B 85, 174424 (2012).

[13] R. Arita, J. Kuneš, A. V. Kozhevnikov, A. G. Eguiluz, and M. Imada, Phys. Rev. Lett. 108, 086403 (2012).

[14] Y. G. Shi, Y. F. Guo, S. Yu, M. Arai, A. A. Belik, A. Sato, K. Yamaura, E. Takayama-Muromachi, H. F. Tian, H. X. Yang, J. Q. Li, T. Varga, J. F. Mitchell, and S. Okamoto, Phys. Rev. B 80, 161104(R) (2009).

[15] S. Calder, V. O. Garlea, D. F. McMorrow, M. D. Lumsden, M. B. Stone, J. C. Lang, J.-W. Kim, J. A. Schlueter, Y. G. Shi, K. Yamaura, Y. S. Sun, Y. Tsujimoto, and A. D. Christianson, Phys. Rev. Lett. 108, 257209 (2012).

[16] D. Khomskii, Physics 2, 20 (2009).

[17] C.-G. Duan, J. P. Velev, R. F. Sabirianov, Z. Zhu, J. Chu, S. S. Jaswal, and E. Y. Tsymbal, Phys. Rev. Lett. 101, 137201 (2008).

[18] G. Kresse, and J. Hafner, Phys. Rev. B 47, R558 (1993).

[19] G. Kresse, and J. Furthmüller, Phys. Rev. B 54, 11169 (1996).

[20] J. P. Perdew, K. Burke, and M. Ernzerhof, Phys. Rev. Lett. 77, 3865 (1996).

[21] See Supplemental Material.

[22] R. E. Cohen, Nature (London) 358, 136 (1992).

[23] N. A. Hill, J. Phys. Chem. B 104, 6694 (2000).

[24] P. Ghosez, J.-P. Michenaud, and X. Gonze, Phys. Rev. B 58, 6224 (1998).

[25] L. F. Mattheiss, Phys. Rev. B 13, 2433 (1976).

[26] I. Inbar, and R. E.Cohen, Phys. Rev. B 53, 1193 (1996).

\section{SUPPLEMENTARY}

In order to investigate the effect of spin-orbital coupling (SOC) on the electronic structures, we compare the results obtained with and without SOC. As demonstrated in Fig. 5, the band structure difference around the Fermi level is negligible.

We also calculate potential energy surface based on experimental displacements [1] by using available exchange- 

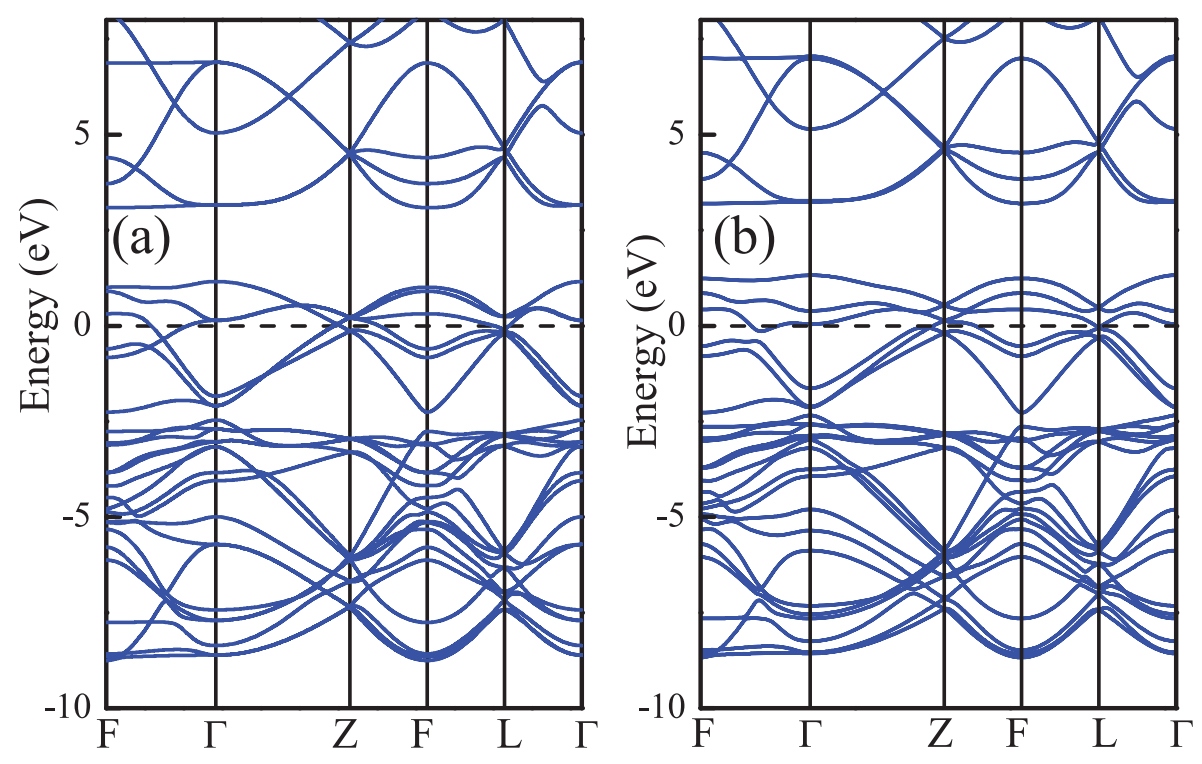

FIG. 5: Band structure of $\mathrm{LiOsO}_{3}$, shown along the high-symmetry directions. (a) GGA, (b) GGA + SO.

correlation functionals (such as: LDA, PBE and PW91) and the results are presented in Fig. 6. In each case, dense $k$ mesh and huge energy cutoff for the basis set are carefully checked for better convergence. Adopting experimental coordinates will obtain unreasonable results, as the well depth caused by the sole Li ion movements is even larger than that of considering both the $\mathrm{Li}$ and $\mathrm{O}$ moments. 
Manuel, Y. Michiue, A. Sato, K. Tsuda, S. Yu, M. Arai, Y. Shirako, M. Akaogi, N. Wang, K. Yamaura and A. T. Boothroyd, Nat. Mat. 12, 1024 (2013).

* Corresponding author: xgwan@nju.edu.cn

[1] Y. Shi, Y. Guo, X. Wang, A. J. Princep, D. Khalyavin, P. 


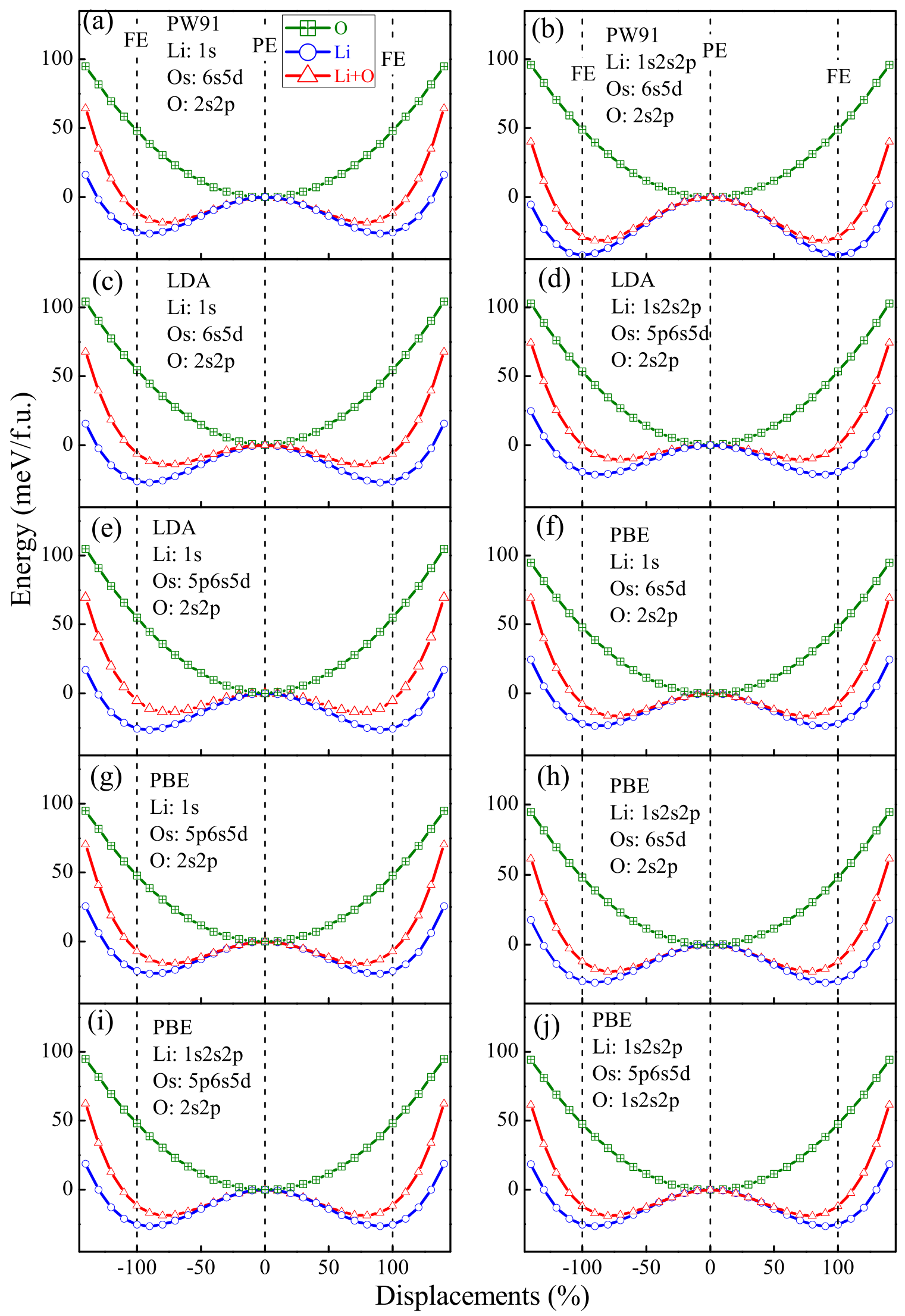

FIG. 6: (Color online)Potential energy surface based on experimental displacements [1] using other available exchangecorrelation functionals: (a)-(b) PW91, (c)-(e) LDA, (f)-(j) PBE. For each exchange-correlation functional, the calculations are performed using pseudopotentials with different valence electrons, and the used valence states for $\mathrm{Li}$, Os and $\mathrm{O}$ are inserted in related figures. The olive, blue and red 